\title{
FILAMENTATIONS FOR VIRTUAL LINKS
}

\author{
WILLIAM J. SCHELLHORN
}

\begin{abstract}
In 2002, D. Hrencecin and L.H. Kauffman defined a filamentation invariant on oriented chord diagrams that may determine whether the corresponding flat virtual knot diagrams are non-trivial (see [2]). A virtual knot diagram is non-classical if its related flat virtual knot diagram is non-trivial. Hence filamentations can be used to detect non-classical virtual knots. We extend these filamentation techniques to virtual links with more than one component. We also give examples of virtual links that they can detect as non-classical.
\end{abstract}

\section{INTRODUCTION}

Virtual links were first introduced by L.H. Kauffman in 1996. A link, in the classical sense, is a special type of virtual link. Hence any results that hold for virtual links must also apply to classical links. This leads to the study of invariants of virtual links. In particular, we are interested in invariants that have the ability to distinguish virtual links that are not classical links. D. Hrencecin and L.H. Kauffman introduced one such invariant on virtual knots using filamentations on oriented chord diagrams (see [2]).

In this paper, we extend the filamentation invariant to virtual links with more than one component. In Section 2, we recall the basic definitions of virtual link theory and indicate how chord diagrams characterize virtual links. In Section 3, we define filamentations on oriented chord diagrams that represent virtual links. The theorems of Section 4 give the central results of this paper. Theorem 4.2 shows that the existence of a filamentation is an invariant of oriented chord diagrams. Theorem 4.3 and Corollary 4.4 then describe how filamentations can be used to distinguish non-classical virtual links. Some additional results and interesting examples are included in Section 5.

Date: November 21, 2003.

1991 Mathematics Subject Classification. 57M27; 57M25.

Key words and phrases. filamentation, virtual link, chord diagram. 


\section{Chord Diagrams}

A link of $n$ components is a subset of $S^{3}$ that consists of $n$ disjoint, piecewise linear, simple closed curves, i.e. the image of a piecewise smooth embedding of $n$ copies of $S^{1}$ into $S^{3}$. In particular, a link of one component is called a knot. A link can be put in general position with respect to the standard projection from $\mathbb{R}^{3}$ to $\mathbb{R}^{2}$, so that the only singularities are transversal double points. A link diagram for a link is the image of the link in $\mathbb{R}^{2}$ enhanced with "over" and "under" crossing information at each double point. Therefore a link diagram is a 4-valent graph in $\mathbb{R}^{2}$ with additional structure associated to its nodes. Two link diagrams are called equivalent if there exists a sequence of Reidemeister moves relating them. A link $L$ can then be defined in a combinatorial sense as an equivalence class of link diagrams for $L$ under the Reidemeister moves. This is the approach we will take in this paper.

A virtual link diagram is also a 4-valent graph in $\mathbb{R}^{2}$ with additonal structure accociated to its nodes. A node in a virtual link diagram can be an over or under crossing as in a link diagram, or it can be a virtual crossing. A virtual crossing is represented in a virtual link diagram as a node with a small circle around it. The over and under crossings of a virtual link diagram are called real crossings to distinguish them from its virtual crossings. Two virtual link diagrams are called equivalent if there exists a sequence of Reidemeister moves and virtual Reidemeister moves relating them. A virtual link $L$ can be defined in a combinatorial sense as an equivalence class of virtual link diagrams for $L$ under the Reidemeister and virtual Reidemeister moves. A detailed introduction to the theory of virtual links is presented in 3. Notice that a link diagram is simply a virtual link diagram with no virtual crossings. Hence link diagrams are referred to as classical link diagrams, and a virtual link is called a classical link if it can be represented by a classical link diagram.

Definition 2.1. A flat virtual link diagram is a 4-valent graph in $\mathbb{R}^{2}$ with nodes that may or may not be virtual crossings. A flat virtual link diagram can be obtained from a virtual link diagram by flattening it, that is, stripping the real crossing information from its nodes.

As described in [2], any virtual link diagram can be characterized by an arrow diagram ${ }^{1}$ and any flat virtual link diagram can be characterized by an oriented chord diagram. We will refer to arrow diagrams as AD's and oriented chord diagrams as OCD's.

\footnotetext{
${ }^{1}$ Such a chord diagram was referred to as a Gauss diagram in $[1]$.
} 


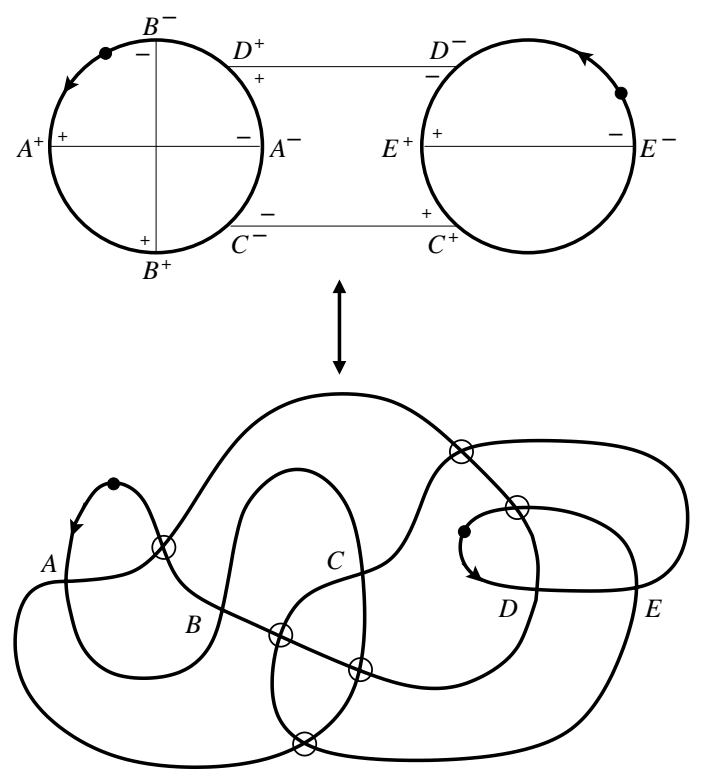

Figure 1. An OCD and its related flat virtual link diagram.

Example 2.2. Figure 1 shows a flat virtual link diagram and its associated OCD.

Example 2.3. Figure 2 shows a virtual link diagram and its associated AD.

The flat Reidemeister moves for flat virtual link diagrams are depicted in Figure 3. These moves are flattened versions of the Reidemeister moves and virtual Reidemesiter moves. Two flat virtual link diagrams are called equivalent if there exists a sequence of flat Reidemeister moves relating them. Then a flat virtual link can be defined as an equivalence class of flat virtual link diagrams under the flat Reidemeister moves.

Definition 2.4. Let $L$ be a virtual link, and let $D$ be the flat virtual link diagram that results from flattening a virtual link diagram for $L$. The universe of $L$ is the flat virtual link represented by $D$.

Example 2.5. The universe of the virtual link in Figure 2 is represented by the flat virtual link diagram in Figure 1,

An equivalence relation can be defined on the set of all OCD's that reflects the flat Reidemeister moves. Notice the flat Reidemeister moves involving virtual crossings do not change the associated OCD since virtual crossings are not represented by chords in the OCD. Figure 4 


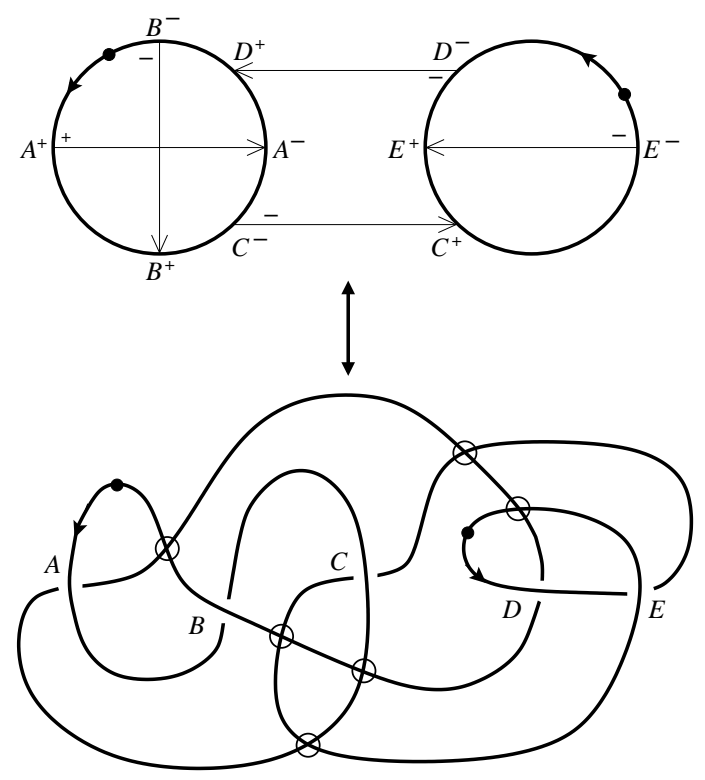

Figure 2. An AD and its related virtual link diagram.
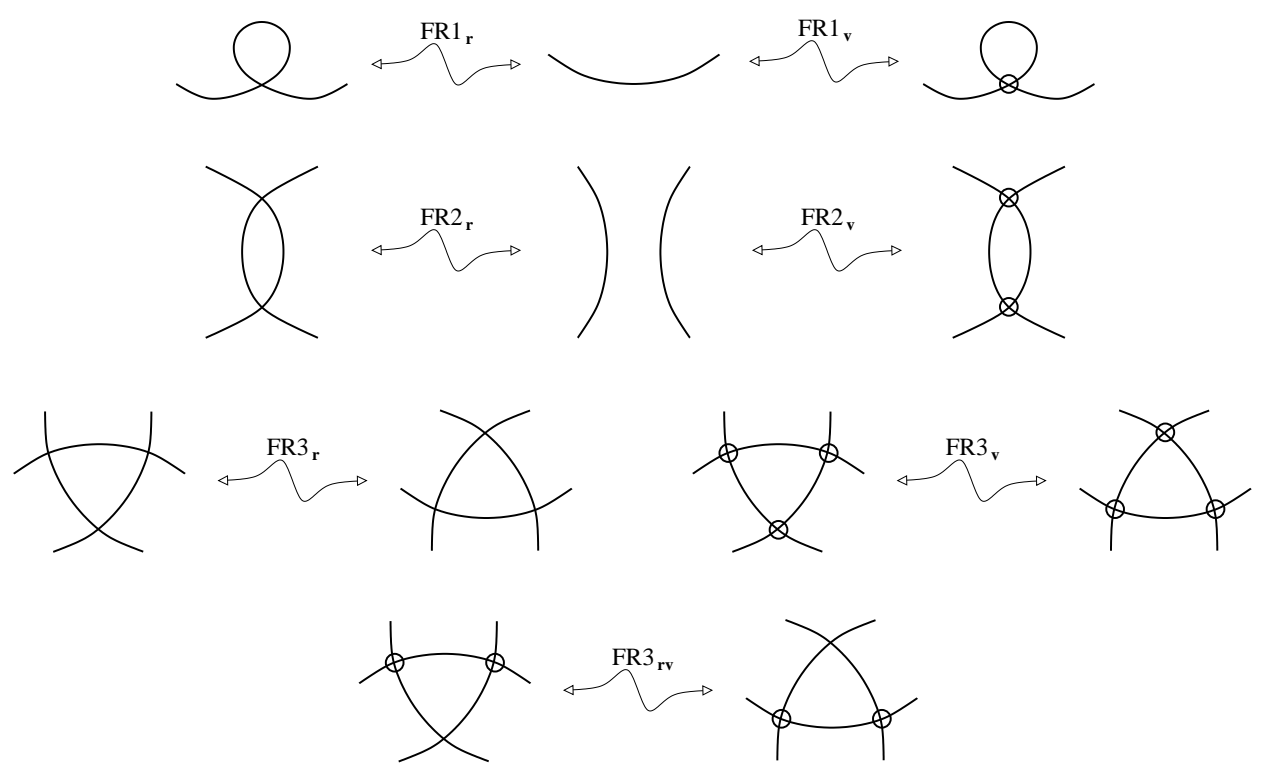

Figure 3. The flat Reidemeister moves.

depicts the OCD moves resulting from the flat Reidemeister moves that do not involve virtual crossings, where the move $\mathrm{OCD}_{i}$ results from the move $\mathrm{FR} i_{\mathrm{r}}$ for $i \in\{1,2,3\}$. Note $\varepsilon$ can be either + or - . The thick arcs depicted are unordered and may be on different circles of an OCD. 


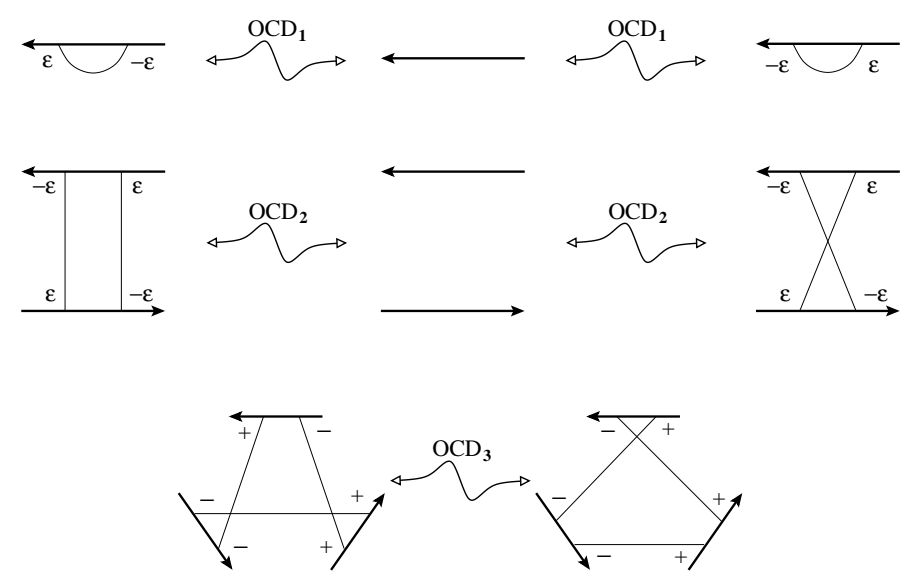

Figure 4. The OCD moves.
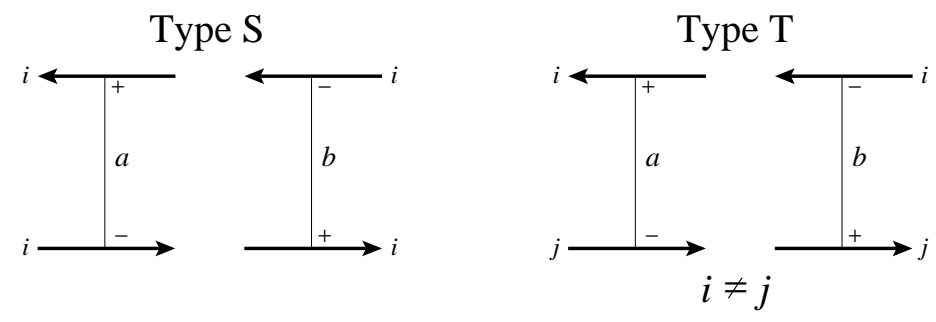

Figure 5. The two types of acceptable chord pairs.

\section{Filamentations}

Definition 3.1. An acceptable chord pair in an oriented chord diagram $D$ is a chord pair $(a, b)$ such that the positive end of $a$ and the negative end of $b$ are on the same circle, and the negative end of $a$ and the positive end of $b$ are on the same circle. We will say the chord pair is of type $S$ if the two circles are the same, and of type $T$ if the two circles are distinct. See Figure 5, where the labels $i$ and $j$ indicate the circle of $D$ on which the chord endpoints reside. Note that it is possible for $a=b$ in a type $\mathrm{S}$ acceptable chord pair, but not in a type $\mathrm{T}$ pair.

Definition 3.2. A pairing $\mathcal{P}$ for a chord diagram $D$ is a collection of chord pairs such that each chord in $D$ occurs in exactly one pair in the collection and each chord pair is acceptable.

Throughout the remainder of this paper, we will use lowercase letters to denote chords and the corresponding uppercase letters with sign indicators to denote the endpoints of chords. 
Example 3.3. The OCD in Figure 1 has these two possible pairings:

$$
\begin{gathered}
\{(a, a),(b, b),(c, d),(e, e)\} \\
\{(a, b),(c, d),(e, e)\}
\end{gathered}
$$

Definition 3.4. Let $D$ be an OCD, and let $(a, b)$ be an acceptable chord pair for $D$. A filament $\alpha$ associated with $(a, b)$ is a generic curve between an endpoint $A^{\varepsilon}$ of $a$ and the endpoint $B^{-\varepsilon}$ of $b$, where $\varepsilon \in\{+,-\}$. Excluding its endpoints, the curve must lie entirely within the interior of a single circle of $D$ and may contain a finite number of transverse self-intersections. A filament is oriented from its negative endpoint to its positive endpoint. The filament between $A^{-\varepsilon}$ and $B^{\varepsilon}$ is called the dual of $\alpha$, and is denoted $\alpha^{\prime}$.

If the chords $a$ and $b$ in this definition are distinct, then the filaments $\alpha$ and $\alpha^{\prime}$ are called bifilaments. Otherwise, $\alpha$ is its own dual, and $\alpha$ is called a monofilament. Note that a filament $\alpha$ and its dual filament $\alpha^{\prime}$ reside on different circles when $(a, b)$ is a type $\mathrm{T}$ chord pair. Therefore filaments coming from type $\mathrm{T}$ chord pairs cannot be monofilaments. We may assume that distinct filaments on an OCD intersect each other transversely. The oriented intersection number $\alpha \vec{\cap} \beta$ of two filaments and the intersection number $\langle\alpha\rangle$ of a filament are defined in [2].

Definition 3.5. Let $(a, b)$ be an acceptable chord pair in a pairing $\mathcal{P}$, and let $\alpha$ be a filament for $(a, b)$. The intersection number of $(a, b)$ is defined as

$$
\langle(a, b)\rangle=\sum_{\beta \in\left\{\alpha, \alpha^{\prime}\right\}} \sum_{\gamma \notin\left\{\alpha, \alpha^{\prime}\right\}} \beta \vec{\cap} \gamma
$$

where the second sum runs over all filaments for the pairs in $\mathcal{P}-\{(a, b)\}$.

Definition 3.6. A filamentation $\mathcal{F}$ on an oriented chord diagram is a pairing for which the related filaments intersect transversely (if they intersect at all) and the intersection number of each pair is zero.

Example 3.7. The OCD in Figure 1 does not admit a filamentation.

Example 3.8. The OCD in Figure 6] admits the filamentation

$$
\{(a, b),(c, d),(e, f)\}
$$

since

$$
\begin{aligned}
& \langle(a, b)\rangle=\langle\alpha\rangle+\left\langle\alpha^{\prime}\right\rangle=-1+1=0, \\
& \langle(c, d)\rangle=\langle\beta\rangle+\left\langle\beta^{\prime}\right\rangle=-1+1=0, \text { and } \\
& \langle(e, f)\rangle=\langle\gamma\rangle+\left\langle\gamma^{\prime}\right\rangle=+1-1=0 .
\end{aligned}
$$




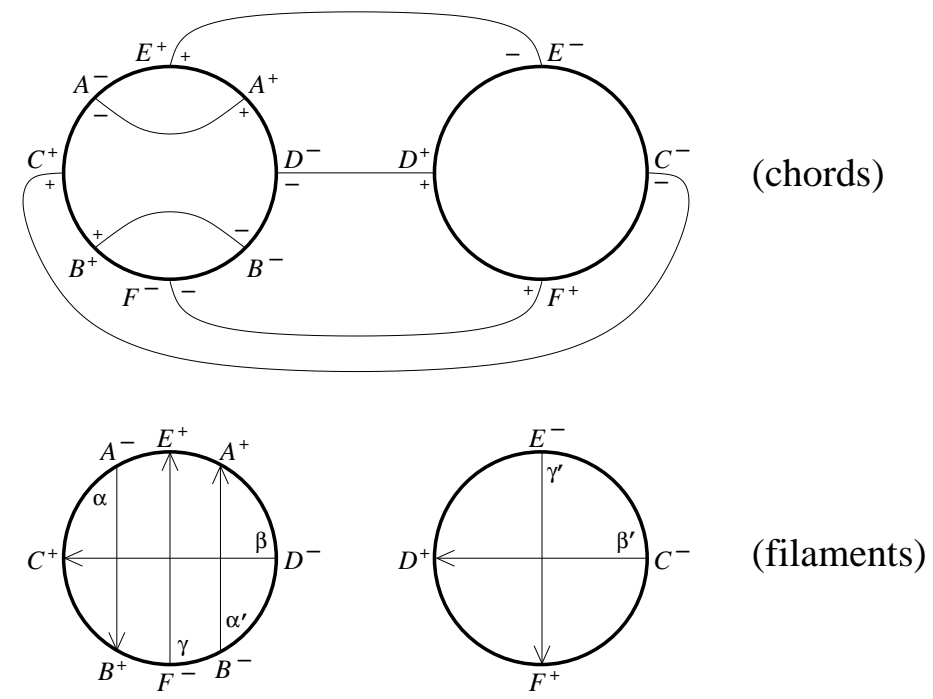

Figure 6. An OCD that admits a filamentation.

\section{THEOREMS}

Theorem 4.1. If a virtual link is classical, then its universe is flat equivalent to the unlink.

Proof. Let $L$ be a classical link. Any classical link can be unlinked via a finite number of crossing changes. So there exists a link diagram for $L$ and a diagram $D_{U}$ for the unlink that share the same universe. There is a finite sequence of Reidemeister moves taking $D_{U}$ to the unlink diagram with no crossings. The corresponding sequence of flat Reidemeister moves will unlink the universe of $L$.

Theorem 4.2. Let $D$ be an $O C D$ for a flat virtual link which admits a filamentation $\mathcal{F}$, and suppose $D^{\prime}$ is a chord diagram equivalent to $D$ via OCD moves. Then there exists a filamentation $\mathcal{F}^{\prime}$ on $D^{\prime}$.

Proof. It suffices to show that under any single OCD move, a filamentation can always be preserved. The result was proved in [2] for cases involving a single circle of an OCD, that is, for knots. Therefore we need only consider cases involving multiple components. We will show how to construct the desired filamentation $\mathcal{F}^{\prime}$ from the existing filamentation $\mathcal{F}$.

In an $\mathrm{OCD}_{1}$ move, both endpoints of the removed or added chord are on the same circle. Consequently, any acceptable chord pair in $\mathcal{F}$ including the chord must be of type S. So either the filament $\alpha$ for this chord pair is a monofilament, or $\alpha$ and its dual filament $\alpha^{\prime}$ are on the 
same circle of $D$. Hence the $\mathrm{OCD}_{1}$ move produces no new cases for our consideration.

There are three new cases to consider for the $\mathrm{OCD}_{2}$ move. The first two, (R1.f) and (R1.g), are depicted in the following diagram ${ }^{2}$ :

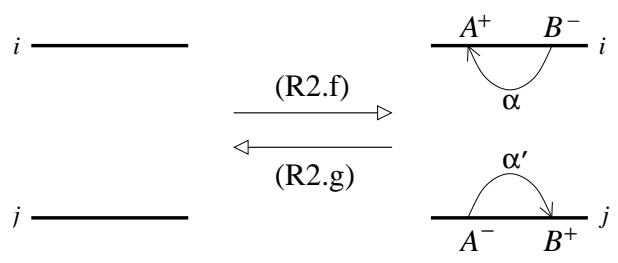

In (R2.f), the induced filamentation $\mathcal{F}^{\prime}$ is defined by adding the type $\mathrm{T}$ pair $(a, b)$ to $\mathcal{F}$, that is $\mathcal{F}^{\prime}=\mathcal{F} \cup\{(a, b)\}$. In (R2.g), remove $(a, b)$ from $\mathcal{F}$ by setting $\mathcal{F}^{\prime}=\mathcal{F}-\{(a, b)\}$. The other case is depicted in the following diagram:



Here we can define $\mathcal{F}^{\prime}=(\mathcal{F}-\{(a, x),(b, y)\}) \cup\{(x, y)\}$, since

$$
\begin{aligned}
\langle(x, y)\rangle_{\mathcal{F}^{\prime}} & =\langle\gamma\rangle+\left\langle\gamma^{\prime}\right\rangle \\
& =\left(\langle\alpha\rangle_{\mathcal{F}}+\left(\left\langle\beta^{\prime}\right\rangle_{\mathcal{F}}\right)+\left(\langle\beta\rangle_{\mathcal{F}}+\left\langle\alpha^{\prime}\right\rangle_{\mathcal{F}}\right)\right. \\
& =\left(\langle\alpha\rangle_{\mathcal{F}}+\left(\left\langle\alpha^{\prime}\right\rangle_{\mathcal{F}}\right)+\left(\langle\beta\rangle_{\mathcal{F}}+\left\langle\beta^{\prime}\right\rangle_{\mathcal{F}}\right)\right. \\
& =0 .
\end{aligned}
$$

Now consider the $\mathrm{OCD}_{3}$ move. As mentioned in [2, for knot OCD's it is not necessary to consider all possible permutations of the circle arcs. The same is true for link OCD's with more than one component by the following argument. We can position the filaments of $\mathcal{F}$ so that none of the intersections occur within small neighborhoods of each circle of $D$. Then filaments for $D^{\prime}$ can be arranged in the same way, except within these neighborhoods the two filaments that emanate from each arc cross. These new crossings on the filaments account for the fact that the $\mathrm{OCD}_{3}$ move simply switches the endpoints on each arc. Refer to Figure 7 for an example. For any possible configuration of an $\mathrm{OCD}_{3}$ move, we define $\mathcal{F}^{\prime}=\mathcal{F}$ and consider the effect of modifying

\footnotetext{
${ }^{2}$ The cases (R1.a) through (R1.e) were discussed in [2] because they concern only virtual knots. We use the letters "f" and "g" here to be consistent with this notational convention.
} 

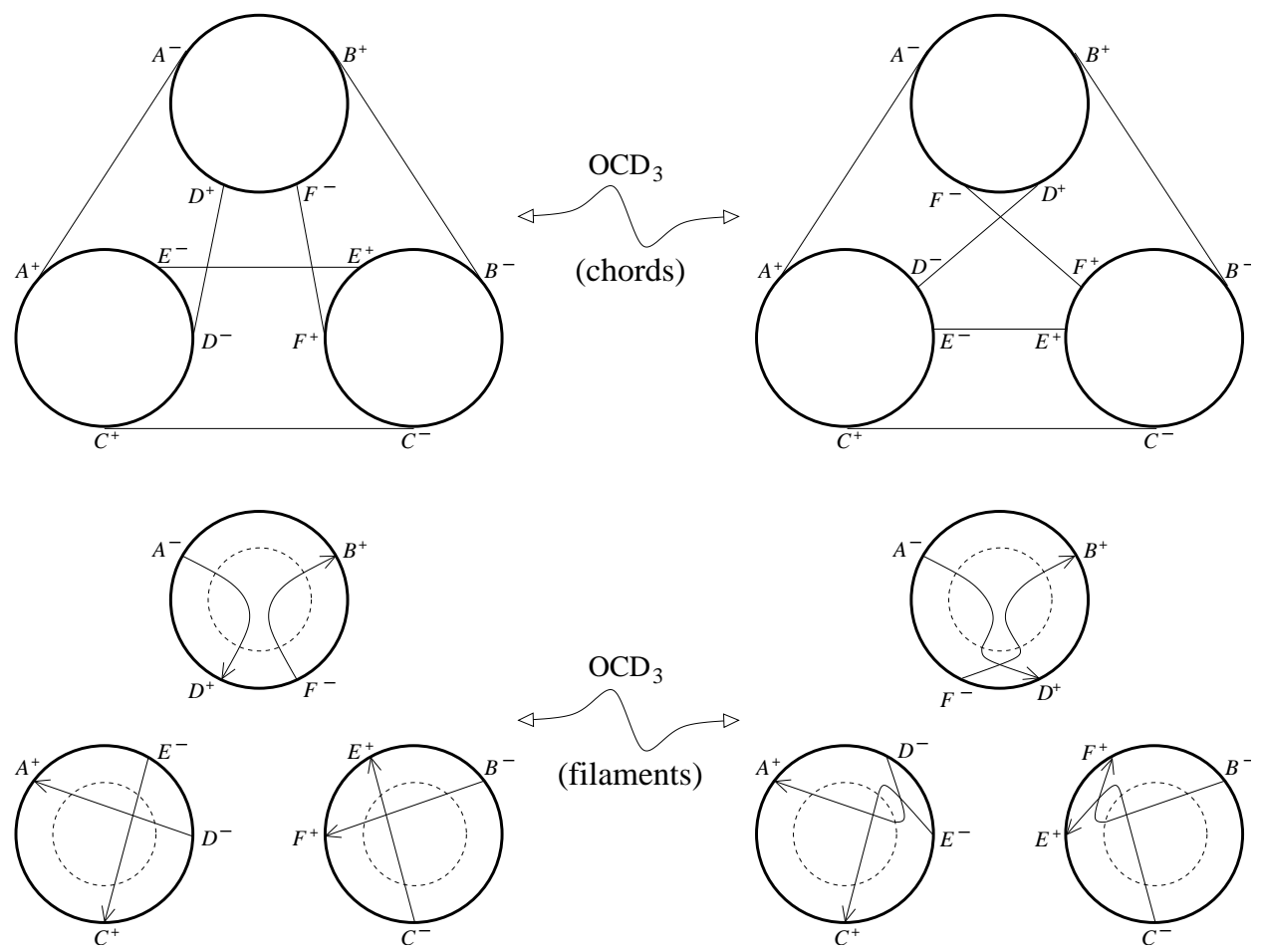

(filaments)
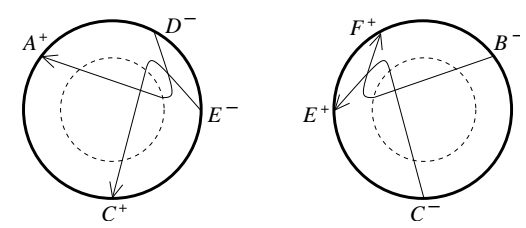

Figure 7 . The effect of an $\mathrm{OCD}_{3}$ move.

the filaments for $D$ to form the filaments for $D^{\prime}$ as described above. In general, we have the situation illustrated in the schematic

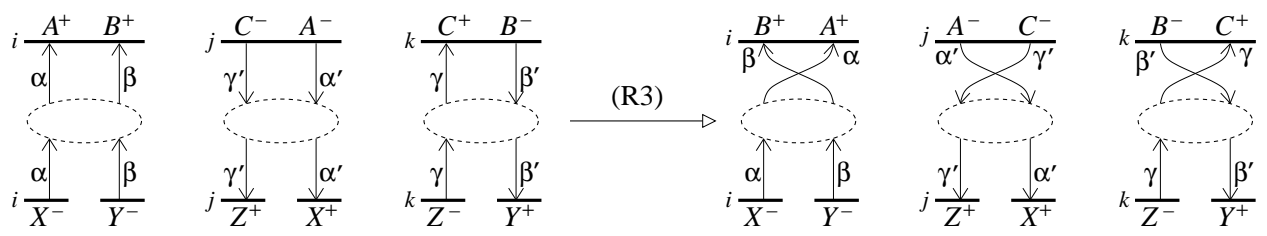

with the following cases:

(1) $i=j \neq k$

(2) $i \neq j=k$

(3) $i=k \neq j$

(4) $i, j, k$ are pairwise distinct

Notice that in any of these four cases, if the depicted filaments $\alpha, \beta$, $\gamma, \alpha^{\prime}, \beta^{\prime}$, and $\gamma^{\prime}$ are all distinct, then

$$
\begin{aligned}
& \langle(a, x)\rangle_{\mathcal{F}^{\prime}}=\langle(a, x)\rangle_{\mathcal{F}}+\alpha \vec{\cap} \beta+\alpha^{\prime} \vec{\cap} \gamma^{\prime}=0+1-1=0 \\
& \langle(b, y)\rangle_{\mathcal{F}^{\prime}}=\langle(b, y)\rangle_{\mathcal{F}}+\beta \vec{\cap} \alpha+\beta^{\prime} \vec{\cap} \gamma=0-1+1=0 \\
& \langle(c, z)\rangle_{\mathcal{F}^{\prime}}=\langle(c, z)\rangle_{\mathcal{F}}+\gamma \vec{\cap} \beta^{\prime}+\gamma^{\prime} \vec{\cap} \alpha^{\prime}=0-1+1=0 ;
\end{aligned}
$$


so under this condition, $\mathcal{F}^{\prime}$ is a filamentation because $\mathcal{F}$ is a filamentation. In cases (2), (3), and (4), the depicted filaments must all be distinct. Therefore to prove the result we now suppose case (1) holds and the filaments are not pairwise distinct. If the pairs $(a, x)$ and $(b, c)$ occur in $\mathcal{F}$, where is $x$ neither $b$ nor $c$, then we have

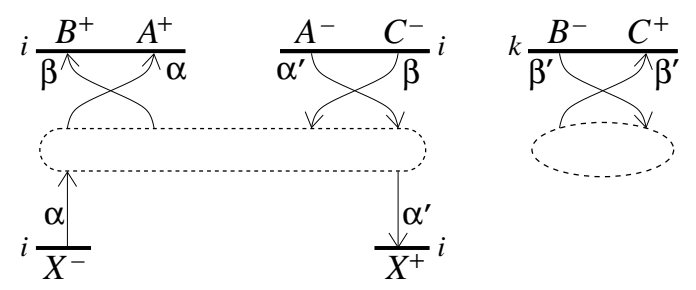

with

$$
\begin{aligned}
\langle(a, x)\rangle_{\mathcal{F}^{\prime}} & =\langle(a, x)\rangle_{\mathcal{F}}+\alpha \vec{\cap} \beta+\alpha^{\prime} \vec{\cap} \gamma^{\prime}=0+1-1=0 \\
\langle(b, c)\rangle_{\mathcal{F}^{\prime}} & =\langle(b, c)\rangle_{\mathcal{F}}+\beta \vec{\cap} \alpha+\beta \vec{\cap} \alpha^{\prime}=0-1+1=0
\end{aligned}
$$

since the self-intersections of $\beta^{\prime}$ do not contribute to $\left\langle\beta^{\prime}\right\rangle$. If the pairs $(a, a)$ and $(b, c)$ occur in $\mathcal{F}$, then we have

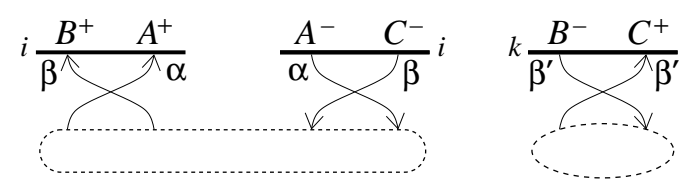

with

$$
\begin{aligned}
\langle(a, a)\rangle_{\mathcal{F}^{\prime}} & =\langle(a, a)\rangle_{\mathcal{F}}+\alpha \vec{\cap} \beta=0+(-1+1)=0 \\
\langle(b, c)\rangle_{\mathcal{F}^{\prime}} & =\langle(b, c)\rangle_{\mathcal{F}}+\beta \vec{\cap} \alpha=0+(1-1)=0
\end{aligned}
$$

since again the self-intersections of $\beta^{\prime}$ do not contribute to $\left\langle\beta^{\prime}\right\rangle$. If the pair $(a, a)$ occurs in $\mathcal{F}$, but not the pair $(b, c)$, then we have

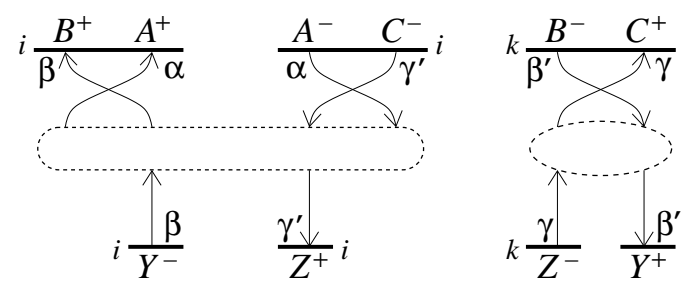

with

$$
\begin{aligned}
& \langle(a, a)\rangle_{\mathcal{F}^{\prime}}=\langle(a, a)\rangle_{\mathcal{F}}+\alpha \vec{\cap} \gamma^{\prime}+\alpha \vec{\cap} \beta=0-1+1=0 \\
& \langle(b, y)\rangle_{\mathcal{F}^{\prime}}=\langle(b, y)\rangle_{\mathcal{F}}+\beta \vec{\cap} \alpha+\beta^{\prime} \vec{\cap} \gamma=0-1+1=0 \\
& \langle(c, z)\rangle_{\mathcal{F}^{\prime}}=\langle(c, z)\rangle_{\mathcal{F}}+\gamma \vec{\cap} \beta^{\prime}+\gamma^{\prime} \vec{\cap} \alpha=0-1+1=0 .
\end{aligned}
$$


Thus we have shown how to construct a filamentation $\mathcal{F}^{\prime}$ on $D^{\prime}$ whenever there exists a filamentation $\mathcal{F}$ on $D$, as desired.

Theorem 4.2 gives that the existence of a filamentation is an invariant of OCD's, and hence an invariant of flat virtual links.

Theorem 4.3. If $D$ is an $O C D$ for which a filamentation does not exist, then the flat virtual link represented by $D$ is non-trivial.

Proof. If $D$ is trivial, then there is a sequence of OCD moves that unlinks $D$. But the unlink admits a trivial filamentation, so by Theorem 4.2. a filamention for $D$ can be constructed.

Corollary 4.4. If $D$ is an $O C D$ for which a filamentation does not exist, then any virtual link with universe represented by $D$ is non-classical.

Proof. The flat virtual link represented by $D$ is non-trivial by Theorem 4.3. Hence the result follows from Theorem 4.1

\section{EXAMPLES}

Definition 5.1. The parity invariant of a flat virtual link (resp. virtual link) is the parity of the total number of either real or virtual crossings between distinct components of the link. Notice that the parity of a flat virtual link (resp. virtual link) is the same as the parity of the total number of chords between distinct components of an OCD (resp. AD) for the link.

Example 5.2. As mentioned in Example 3.7, the OCD in Figure 1does not admit a filamentation, so any virtual link with universe represented by this chord diagram is non-classical. Hence the virtual link in Figure 2 is a non-classical virtual link with two classical components (both unknotted). Moreover, the parity invariant cannot distinguish it from the unlink since it has two real crossings between its two components.

The previous example illustrates that the converse of the following proposition does not hold.

Proposition 5.3. If a flat virtual link $L$ has odd parity and $D$ is an $O C D$ representing $L$, then $D$ does not admit a filamentation.

Proof. Since $L$ has odd parity, it follows that the total number of chords between distinct components of $D$ is odd. Then no pairing exists for $D$ since two such chords must be paired together to form an acceptable chord pair. Hence $D$ cannot admit a filamentation. 


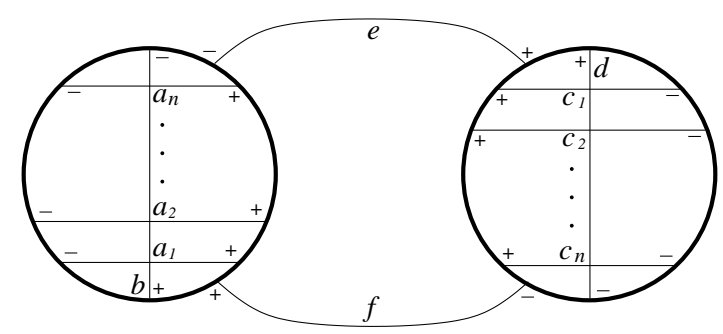

FiguRE 8. The OCD of a non-trivial flat virtual link with two non-trivial components.

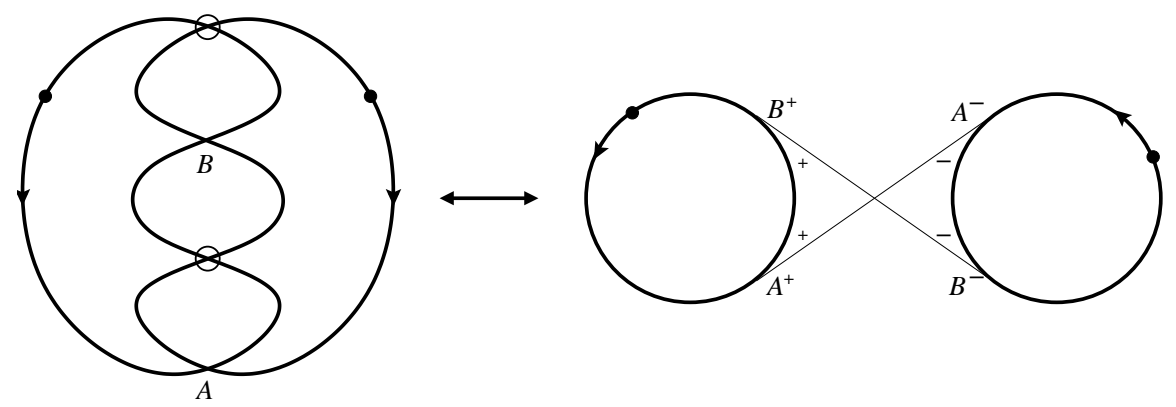

FiguRE 9. A non-trivial flat virtual link with two trivial components and its related OCD.

Example 5.4. Fix $n$ and let $\mathcal{P}$ be a pairing for the OCD in Figure 8 , Then $\mathcal{P}$ must contain the type T pair $(e, f)$, for neither $e$ nor $f$ can be paired with any other chord. Notice $\langle(e, f)\rangle=2 n \neq 0$, so this OCD does not admit a filamentation. Hence any virtual link $L$ with universe represented by this OCD is non-classical. However, the parity invariant is unable to detect this. Each component of $L$ is itself a non-classical knot, as shown in [2].

Example 5.5. The flat virtual link in Figure 9] was given in [2] as an example of a non-trivial flat virtual link that can be detected by a certain specialization of the Alexander Biquandle. Note that the parity invariant fails at this task. However, since its related OCD does not admit any pairings, the filamentation invariant succeeds in detecting that it is linked.

\section{ACKnowledgements}

I wish to thank my advisor R.A. Litherland for the many valuable conversations and suggestions. 


\section{REFERENCES}

[1] M. Goussarov, M. Polyak, and O. Viro, "Finite Type Invariants of Classical and Virtual Knots", preprint - arXiv:math.GT/9810073 1999.

[2] D. Hrencecin and L.H. Kauffman, "On Filamentations and Virtual Knots", preprint - arXiv:math.GT/0112206 2002.

[3] L.H. Kauffman, "Virtual Knot Theory", European J. Comb., 20:663-690, 1999.

Department of Mathematics, Louisiana State University, Baton Rouge, LA 70803-4918

E-mail address: schellho@math.1su.edu 